\title{
Etching away at the surface
}

Silver-based nanoparticles in combination with an etching procedure facilitate the analysis of cellular internalization processes.

Nanoparticles have emerged as useful tools in a variety of biological applications, from molecular imaging to drug delivery. Because most drug targets are localized intracellularly, it is important to monitor the efficiency of nanoparticle uptake into cells. For quantification, extracellularly and intracellularly located nanoparticles need to be distinguished. Erkki Ruoslahti and his staff scientist Gary Braun at the SanfordBurnham Medical Research Institute have been applying nanoparticles to their studies of tumor-specific metastasis. In recent work, they developed nanoparticles that are specifically taken up by tumor cells and paired these particles with an efficient protocol to remove them from the surface of cells.

Ruoslahti's team uses silver nanoparticles coated with both fluorescent dyes and specific targeting peptides to study cellular internalization. The close proximity of the fluorescent dyes to the metal surface leads to a plasmonic effect, which enhances the fluorescence of the dyes. "The interaction of light with metal structures causes certain resonances with the electromagnetic field, focusing the energy just outside the structure, precisely where the dyes are positioned," explains Braun. "The fluorescent dyes appear about tenfold more than their natural brightness when there is no metal." The effect arises with a variety of dyes in the visible spectrum, making the nanoparticles ideal for multispectral applications.

In the field of nanomedicine, "drug carrier internalization is key to maximizing the dose versus off-target effects and to minimize toxicity," says Braun. To study peptidemediated uptake of nanoparticles into cells, it is important to quantify internalized particles. The researchers therefore developed a redox-based destain solution that selectively dissolves any silver nanoparticles on the outside of the cell membrane but does not disrupt the cell membrane. The etchant is mild enough not to impair cell viability in culture, at least when applied for a short time.

The researchers demonstrated the usefulness of their nanoparticles and the etching procedure by imaging nanoparticles in the endocytic pathway in live tumor cells.

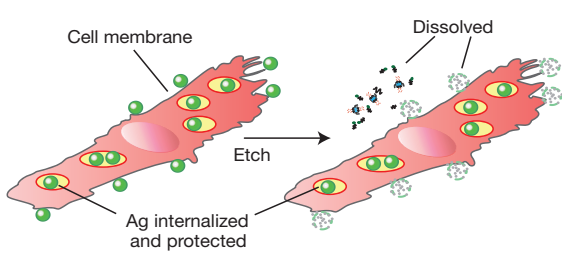

Etching of silver (Ag) nanoparticles selectively retains internalized nanoparticles. Figure from Braun et al., Nature Publishing Group.

Ruoslahti and his team managed to target nanoparticles to cells and then track them for more than 25 minutes inside cells, a feat requiring bright and photostable nanoparticles. In addition, the fluorescent nanoparticles, when decorated with tumor-penetrating peptides, can infiltrate tumor slices; the etching procedure subsequently reveals internalized particles. This enabled the researchers to localize hotspots for endocytosis in the tissue.

The etching process is also applicable to studies in live mice, without adverse effects. "The individual chemicals in the etching solution have been previously used in vivo for various purposes," notes Ruoslahti. To determine the uptake efficiency of nanoparticles, the researchers first injected nanoparticles functionalized with tumor-penetrating peptides into a live mouse tumor model and then later injected the etching solution into the bloodstream. With this technique, Ruoslahti and his team observed $25 \%$ internalization rates.

Etchable nanoparticles will allow a variety of questions concerning internalization processes to be addressed. Ruoslahti is currently using these nanoparticles to study the endocytic transport pathway that mediates tumor penetration of peptides as well as in vivo peptide homing. Although Ruoslahti considers the etchable nanoparticles mainly as research tools, he imagines that they may have therapeutic applications as well. "If we can target the silver nanoparticles to a site of infection with a sufficient efficiency, we might be able to overcome the toxicity of systemically administered silver," he notes, which would allow the dissolved silver to be used as a local antibacterial agent.

Nina Vogt

RESEARCH PAPERS

Braun, G.B. et al. Etchable plasmonic nanoparticle probes to image and quantify cellular internalization. Nat. Mater. doi:10.1038/nmat3982 (8 June 2014). 